\title{
Assessment of Multiple Intravenous Pumps Infusing into a Single Site
}

Nancy Ribeiro, RTNM, Maria Green, RTNM, and Martin Charron, MD

Department of Diagnostic Imaging, Hospital for Sick Children, Toronto, Canada

Much research has examined drug concentration and flow rates for a single intravenous pump infusing into a single site, but there is no information on the delivery of medications when a multiplepump system is required. The intent of this study was to evaluate the accuracy of multiple-pump systems infusing into a single site. We noted large fluctuations when multiple intravenous pumps infused into a single site.

Key Words: delivery pumps; pediatric; instrumentation

J Nucl Med Technol 2008; 36:88-90

DOI: 10.2967/jnmt.107.049197

\footnotetext{
C
}

itically ill patients receive concurrent administration of vasoactive, arrhythmic, inotropic, sedative, analgesic, and other medications intravenously. Many of these medications have short half-lives, and thus it is necessary to infuse them continuously using infusion pumps. Additionally, precise control of the delivery of some of these powerful medications is essential to avoid fluctuations in the patient's clinical condition and to achieve the desired therapeutic endpoints. However, the population of critically ill patients frequently presents with significant limitations in intravenous access because of vein fragility and a poor general condition. Many invasive methods such as peripherally inserted central catheters, central venous lines, and ports are commonly used in these patients to obtain at least 1 infusion site, often resulting in the use of this single line for multiple infusions. In this report, we describe our evaluation of the accuracy of multiple pumps infusing into a single site.

\section{MATERIALS AND METHODS}

A $74 \mathrm{MBq} / \mathrm{mL}$ solution of sodium pertechnetate $\left(\mathrm{NaTcO}_{4}\right)$ in $20 \mathrm{~mL}$ of saline was mixed in a $60-\mathrm{mL}$ syringe and flushed through two 1.5 -mL fluid capacity lines connected via a high-flow 4-way stopcock. The 60 -mL syringe was connected to an IVAC 3000P syringe pump (Cardinal Health). According to the manufacturer of the pump, its system accuracy has been determined to

Received Nov. 16, 2007; revision accepted Mar. 10, 2008.

For correspondence or reprints contact: Martin Charron, Hospital for Sick Children, 555 University Ave., Toronto, Ontario, Canada M5G 1X8.

E-mail: martin.charron@sickkids.ca

COPYRIGHT @ 2008 by the Society of Nuclear Medicine, Inc. be $\pm 2 \%$ at infusion rates of $1.0 \mathrm{~mL} / \mathrm{h}(1)$. Four independent trials were run, in which additional syringe pumps containing $20 \mathrm{~mL}$ of saline in a $60-\mathrm{mL}$ syringe were added, 1 at a time, until the number of pumps totaled 5 (Fig. 1).

Attached to these syringes were $1.5-\mathrm{mL}$ fluid capacity lines. High-flow 4-way stopcocks were piggybacked to connect the

\section{A}

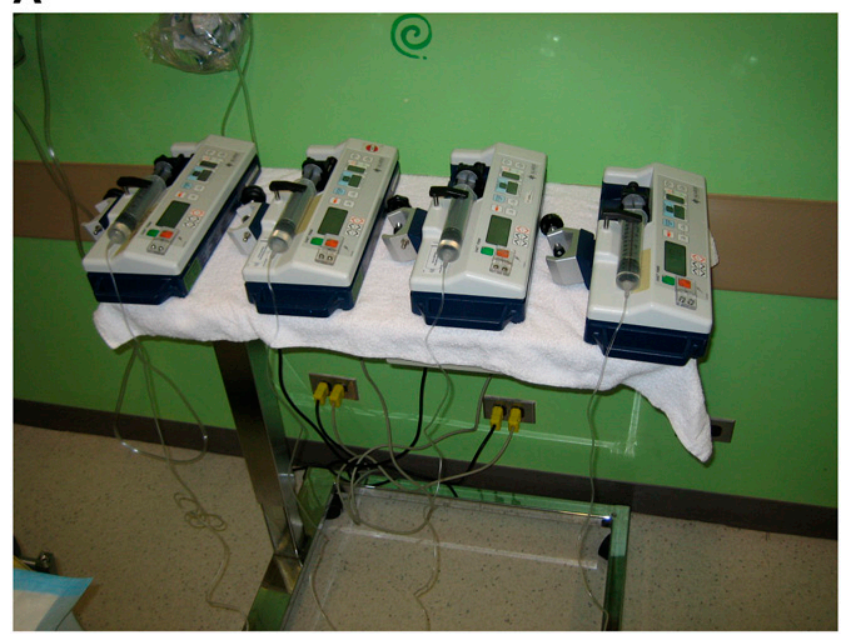

B

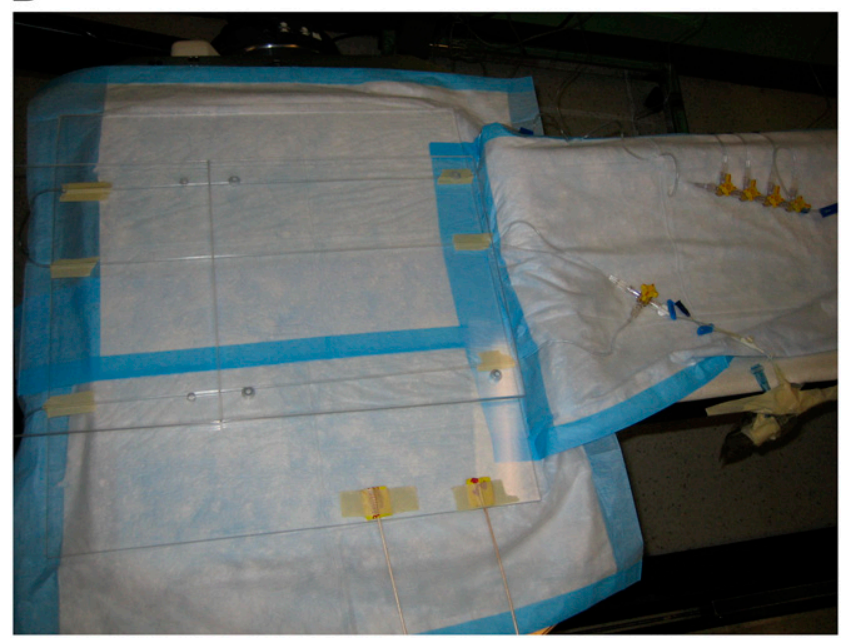

FIGURE 1. Experimental setup for 4-pump system. Shown are the 4 pumps (A) and the 4 lines (B). Similar setups were used for 2-, 3-, and 5-pump systems. All IVAC 3000P syringe pumps were maintained on the same horizontal plane to ensure that gravity did not affect infusion rates. 
additional pumps to the line containing the radioactive solution. In each of the 4 trials, the pump containing the radioactive solution ran, independently, at $1 \mathrm{~mL} / \mathrm{h}$ for $30 \mathrm{~min}$. At $30 \mathrm{~min}, 1,2,3$, or 4 additional pumps containing saline were activated and left to run at $1 \mathrm{~mL} / \mathrm{h}$ for $5.5 \mathrm{~h}$. Imaging was performed using a single-head STAR3000 $\gamma$-camera (GE Healthcare) at $1 \mathrm{~min} /$ frame.

Regions of interest were drawn on the fluid capacity lines where all solutions merged. The obtained values were decaycorrected and used to calculate the largest fluctuation using maximum and minimum observed values and presented as a percentage fluctuation. An F test was also performed at both the $95 \%$ and the $99 \%$ confidence intervals to examine whether differences in counts between a single-pump system and a multiplepump system were statistically significant. To make the mean values and variances comparable, we multiplied the results for the multiple-pump systems by their respective dilution factors. Total activity used for the 2-pump system was lower than that used in the other 3 trials.

An additional 1.5 -mL fluid capacity line flushed with the same radioactive solution was left stationary and imaged concomitantly, serving as a steady-state line to evaluate the accuracy of the apparatus. The accuracy of the multiple-pump systems was assessed by evaluating the variability in counts for a single pump infusing for the first $30 \mathrm{~min}$ into a 2-, 3-, or 4-pump system. The accuracy of the apparatus was assessed by comparing the variability in the counts for 1 pump running for 30 min with the same apparatus running additional pumps after this initial $30 \mathrm{~min}$ (Fig. 2).

\section{RESULTS}

The variability in the concentration of counts observed for the steady-state line was less than $3 \%$. The variability in counts for 1 pump infusing for the initial $30 \mathrm{~min}$ in an apparatus of $2,3,4$, or 5 pumps ranged from $2.9 \%$ to $3.9 \%$ (Table 1).

Figure 2 shows, for the 2- to 5-pump systems, infusion data for a single pump active for the first $30 \mathrm{~min}$ followed
TABLE 1

Data on Largest Fluctuations

\begin{tabular}{llr}
\hline Trial no. & Number of pumps & Largest fluctuation (\%) \\
\hline \multirow{2}{*}{1} & 1 pump; initial 30 min & 3.9 \\
& 2 pumps & 16.6 \\
2 & 1 pump; initial 30 min & 3.5 \\
& 3 pumps & 15.7 \\
3 & 1 pump; initial 30 min & 3.6 \\
& 4 pumps & 15.7 \\
4 & 1 pump; initial 30 min & 2.9 \\
& 5 pumps & 16.9
\end{tabular}

Fluctuation for a single pump running $30 \mathrm{~min}$ in each trial of 2-5 pumps ranged from $2.9 \%$ to $3.9 \%$. This fluctuation increased significantly when 1 or more pumps were added in each trial (15.7\%-16.9\%).

by the addition of 1-4 pumps beyond the first $30 \mathrm{~min}$. Fluctuations increased significantly after additional pumps were added-that is, after $30 \mathrm{~min}$ (range, 15.7\%-16.9\%; $P<0.01)$.

\section{DISCUSSION}

Continuous medication delivery is common in critical care units to maintain patient stability. The precise delivery of many intravenous medications is essential because many of the infused drugs have short half-lives in the circulation. Also, some delivered medications are quite potent, and small fluctuations in the amounts delivered may have unfavorable effects. For example, a bolus delivery of vasoconstrictors may not affect relatively healthy patients but would have a catastrophic effect on patients with failing ventricles or aneurysms.

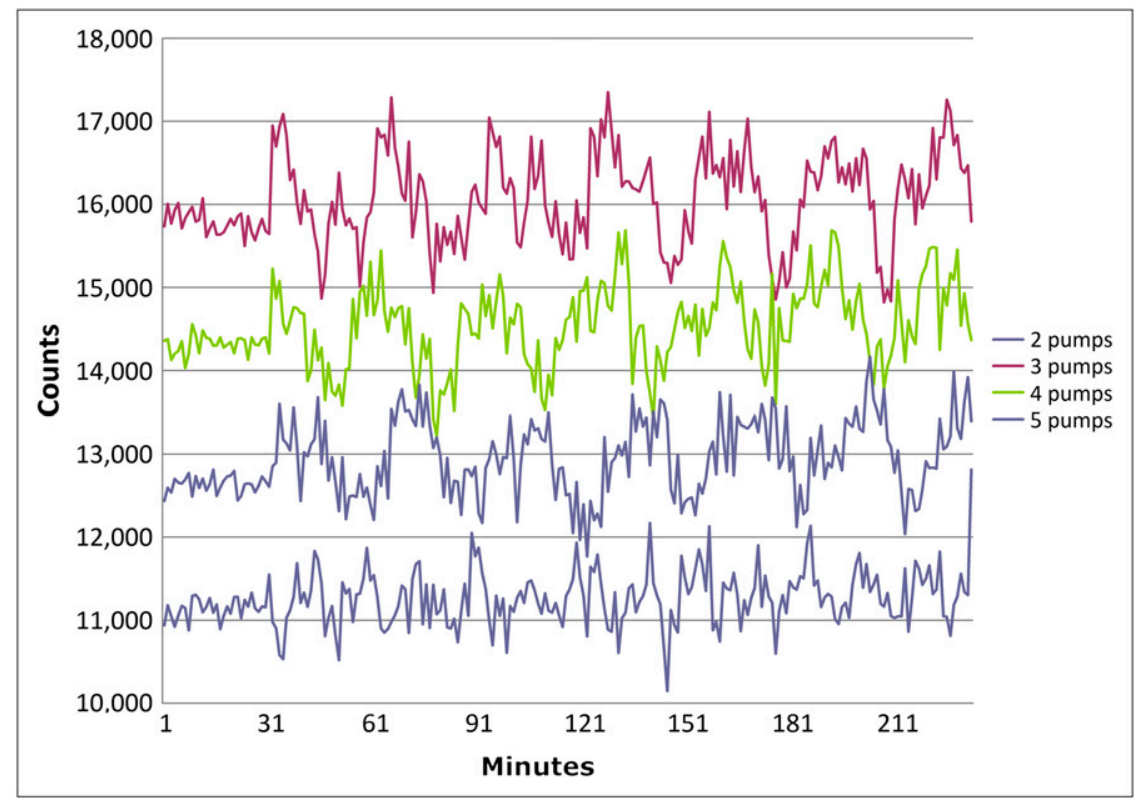

FIGURE 2. Graph of infusion data. Each line represents 1 pump activated for the initial $30 \mathrm{~min}$, immediately followed by activation of the additional pump or pumps. Fluctuations increase as the additional pumps are added. 
Our experimental model demonstrated that adding 1 or more pumps to a single infusion site results in statistically significant fluctuations in the temporal concentration of a radioisotope solution. The volumetric system accuracy of the pump used in our experimental setup was determined by the manufacturer to be $\pm 2 \%$ for a single pump infusing in a single site at a rate of $1 \mathrm{~mL} / \mathrm{h}(1)$. This accuracy held true for each trial when only 1 pump was active, but fluctuations of up to $16.9 \%$ were observed when additional pumps were added (Table 1). To the best of our knowledge, this is the first report of these inaccuracies in the clinical use of this medical device. Currently, the U.S. Food and Drug Administration and Health Canada do not require that manufacturers evaluate the accuracies of multiple-pump systems infusing into a single site. Our data militate for a more stringent regulatory assessment of the uses of pumps in humans. Such assessments may entail designing more accurate pumps or allowing only 1 pump to be used in each infusion site. Alternatively, one could use existing pumps with an awareness of this poor accuracy and, thus, with limitation of their use to only those medications considered safe within the observed range of fluctuation. Further investigation may take into account fluctuations due to gravity as a result of vertical displacement of the infusion pumps and fluctuations when using different types of infusion pumps, different speeds of delivery, and different syringe sizes.

A control line was used in our apparatus to evaluate inaccuracies secondary to, first, statistical counting fluctuations inherent in our tracer (i.e., inherent in radioisotopes) and, second, the $\gamma$-camera. The count fluctuations were higher in the static control line than in the 1-pump system but were much less than in the multiple-pump systems - that is, $7 \%$ in the control line versus $16.9 \%$ in multiple-pump systems.

\section{CONCLUSION}

Multiple pumps infusing into a single site result in a delivery output that is significantly more inaccurate than that specified by the manufacturer for a single pump.

\section{ACKNOWLEDGMENTS}

We thank Chris Parusham and Sanjee Bhargava for their technical contribution. This study was supported by the Department of Radiology of the Hospital for Sick Children.

\section{REFERENCE}

1. IVAC P3000 syringe pump [package insert]. Dublin, OH: Cardinal Health; 2001. 